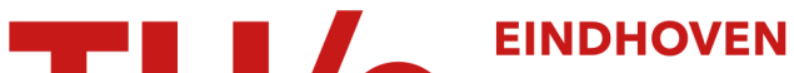 \\ UNIVERSITY OF \\ TECHNOLOGY
}

\section{Microscopic model for all optical switching in ferromagnets}

Citation for published version (APA):

Cornelissen, T. D., Cordoba Castillo, R. M., \& Koopmans, B. (2016). Microscopic model for all optical switching in ferromagnets. Applied Physics Letters, 108(14), 1-4. [142405]. https://doi.org/10.1063/1.4945660

DOI:

$10.1063 / 1.4945660$

Document status and date:

Published: 06/04/2016

\section{Document Version:}

Publisher's PDF, also known as Version of Record (includes final page, issue and volume numbers)

\section{Please check the document version of this publication:}

- A submitted manuscript is the version of the article upon submission and before peer-review. There can be important differences between the submitted version and the official published version of record. People interested in the research are advised to contact the author for the final version of the publication, or visit the $\mathrm{DOI}$ to the publisher's website.

- The final author version and the galley proof are versions of the publication after peer review.

- The final published version features the final layout of the paper including the volume, issue and page numbers.

Link to publication

\section{General rights}

Copyright and moral rights for the publications made accessible in the public portal are retained by the authors and/or other copyright owners and it is a condition of accessing publications that users recognise and abide by the legal requirements associated with these rights.

- Users may download and print one copy of any publication from the public portal for the purpose of private study or research.

- You may not further distribute the material or use it for any profit-making activity or commercial gain

- You may freely distribute the URL identifying the publication in the public portal.

If the publication is distributed under the terms of Article 25fa of the Dutch Copyright Act, indicated by the "Taverne" license above, please follow below link for the End User Agreement:

www.tue.nl/taverne

Take down policy

If you believe that this document breaches copyright please contact us at:

openaccess@tue.nl

providing details and we will investigate your claim. 


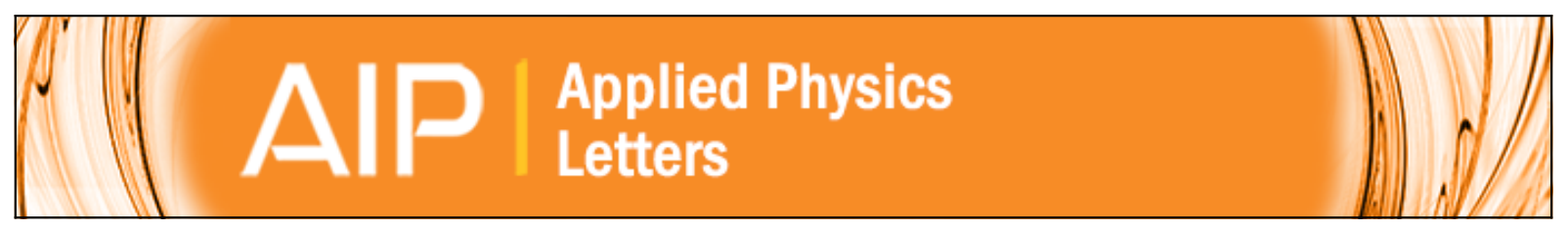

\section{Microscopic model for all optical switching in ferromagnets}

T. D. Cornelissen, R. Córdoba, and B. Koopmans

Citation: Applied Physics Letters 108, 142405 (2016); doi: 10.1063/1.4945660

View online: http://dx.doi.org/10.1063/1.4945660

View Table of Contents: http://scitation.aip.org/content/aip/journal/apl/108/14?ver=pdfcov

Published by the AIP Publishing

\section{Articles you may be interested in}

Electrical characterization of all-optical helicity-dependent switching in ferromagnetic Hall crosses

Appl. Phys. Lett. 108, 092405 (2016); 10.1063/1.4943107

Magnetoresistance of heavy and light metal/ferromagnet bilayers

Appl. Phys. Lett. 107, 192405 (2015); 10.1063/1.4935497

Effect of asymmetric leads on critical switching current in magnetic nanopillars

Appl. Phys. Lett. 89, 082513 (2006); 10.1063/1.2336719

Wavelength dependence of the magnetic resolution of the magneto-optical near-field scanning tunneling microscope

Appl. Phys. Lett. 73, 2669 (1998); 10.1063/1.122548

A combined magneto-optic magnetic force microscope study of $\mathrm{Co} / \mathrm{Pd}$ multilayer films

J. Appl. Phys. 81, 3846 (1997); 10.1063/1.364729

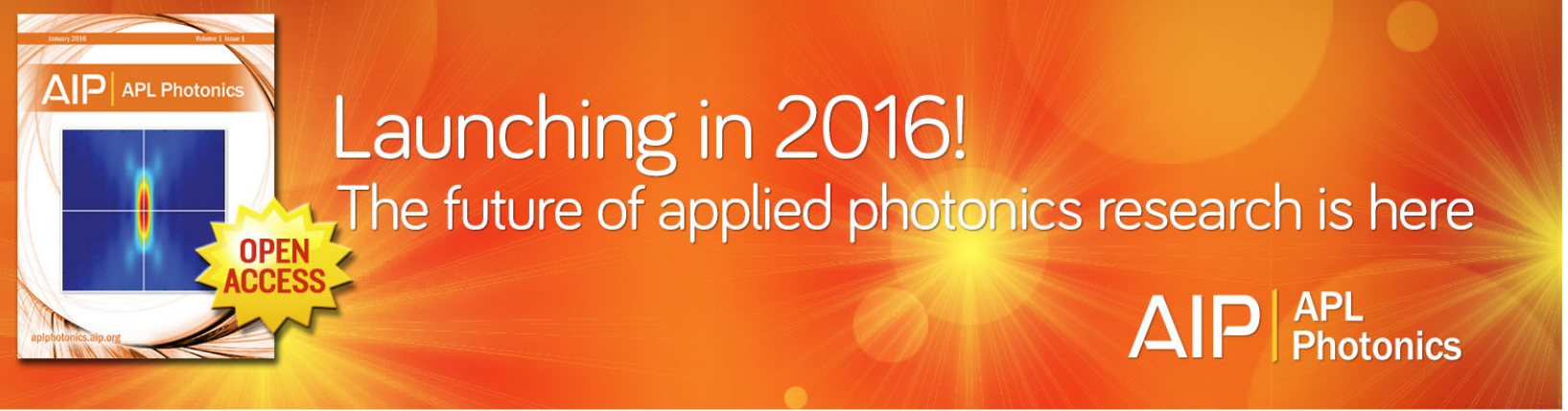




\title{
Microscopic model for all optical switching in ferromagnets
}

\author{
T. D. Cornelissen, R. Córdoba, and B. Koopmans ${ }^{\text {a) }}$ \\ Department of Applied Physics, Center for NanoMaterials, Eindhoven University of Technology, \\ P. O. Box 513, 5600 MB Eindhoven, The Netherlands
}

(Received 31 January 2016; accepted 20 March 2016; published online 6 April 2016)

\begin{abstract}
The microscopic mechanism behind the all optical switching (AOS) in ferromagnets has triggered intense scientific debate. Here, the microscopic three-temperature model is utilized to describe AOS in a perpendicularly magnetized ferromagnetic Co/Pt system. We demonstrate that AOS in such a ferromagnet can be explained with the Inverse Faraday Effect (IFE). The influence of the strength and lifetime of the IFE induced field pulse on the switching process are investigated. We found that because of strong spin-orbit coupling, the minimal lifetime of the IFE needed to obtain switching is of the order of $0.1 \mathrm{ps}$, which is shorter than previously assumed. Moreover, spatial images of the domain pattern after AOS in $\mathrm{Co} / \mathrm{Pt}$, as well as their dependence on applying an opposite magnetic field, are qualitatively reproduced. @ 2016 AIP Publishing LLC.

[http://dx.doi.org/10.1063/1.4945660]
\end{abstract}

The ultrafast manipulation of magnetism using light has received a lot of attention ever since the pioneering experiments of Beaurepaire et al. ${ }^{1}$ in 1996. It has been shown that a $3 \mathrm{~d}$ ferromagnet partially demagnetizes at a fs-time scale upon fs pulsed laser excitation, after which the magnetization recovers at a ps-time scale. This subject is attractive both from a fundamental physics point of view as well as for its technological relevance. One of the most promising applications of the ultrafast optical manipulation of magnetism is to exploit all optical switching (AOS) for magnetic data storage, by using laser pulses to rapidly switch the magnetization. This phenomenon was first discovered in a ferrimagnetic GdFeCo alloy, ${ }^{2}$ and later it was shown to occur in a wider range of materials, including rare-earth-free multilayered synthetic ferrimagnets. ${ }^{3-6}$ However, in all reports AOS was limited to systems with two different sublattices that are coupled antiferromagnetically. Several mechanisms have been proposed to explain AOS in such systems. The (polarized) laser pulse was thought to have a twofold effect. First, it heats up the spin system into a demagnetized and transient ferromagnetic state ${ }^{7}$ a process initially considered independent of its circular polarization. Second, it induces an effective magnetic field by means of the Inverse Faraday Effect (IFE) ${ }^{2,8-11}$ The direction of this field, and thus the direction of remagnetization, is determined by the helicity of the light. ${ }^{2}$ However, later it was found that the helicity dependence was merely a secondary effect and AOS was explained as a result of angular momentum transfer between the two ferrimagnetic sublattices. ${ }^{12}$ Recently, Lambert et al. experimentally demonstrated AOS in simple ferromagnetic structures such as $\mathrm{Co} / \mathrm{Pt}$ multilayers and partial switching in FePt granular films ${ }^{13}$-both of particular relevance for technological applications in data storage and magnetic memory. However, the mechanism behind AOS in ferromagnets remained a mystery, as there are no antiferromagnetically coupled sublattices in these systems. Thus, one of the most viable explanations for AOS could be again the IFE, even though its origin in metals is not well understood

\footnotetext{
${ }^{\text {a) }}$ Author to whom correspondence should be addressed. Electronic mail: b.koopmans@tue.nl
}

on the fundamental level ${ }^{14-19}$ (unlike for example in magnetic semiconductors, where it has been proven experimentally ${ }^{20}$ ). It has also been suggested that dipole interactions between neighboring magnetic domains could play a role in the switching process. ${ }^{21,22}$

Several approaches have successfully accounted for all-optical switching following ultrafast demagnetization. Mentink et al. treated the multi-sublattice switching mode in a general theoretical framework. ${ }^{23}$ In another class of models either the Landau-Lifshitz-Gilbert (LLG) or Landau-LifshitzBloch (LLB) equation is coupled to the two-temperature model for the electron and lattice systems, ${ }^{1}$ to explain AOS in ferrimagnetic $\mathrm{GdFeCo}$ alloy ${ }^{24}$ and the ultrafast demagnetization in magnetic bulk $\mathrm{FePt}^{25}$ and thin films. ${ }^{26}$ Finally, the socalled microscopic three-temperature model (M3TM) applied to multiple spin systems ${ }^{27}$ successfully reproduced the two possible demagnetization behaviors of nickel, ${ }^{28}$ as well as the AOS of ferrimagnetic GdFeCo alloys. ${ }^{29}$

In the present letter, we employ the M3TM to describe AOS in ferromagnets, taking the $\mathrm{Co} / \mathrm{Pt}$ system as a specific model system. We demonstrate that AOS is indeed feasible, using the IFE as the driving mechanism. In addition, nonlocal effects such as dipole fields are also introduced into the model to accurately reproduce AOS images obtained in experiments on ferromagnets. ${ }^{13}$

The M3TM uses a microscopic Hamiltonian to describe the electron, phonon, and spin system and their interactions. It assumes spin-less free electrons (e), phonons according to the Einstein or Debye model (p), and spin excitations obeying the mean-field Weiss model (s). It explicitly models transfer of angular momentum between the electron, lattice, and spin system by means of an Elliott-Yafet type of spin scattering. A Gaussian profile of the laser pulse (Gaussian width $\sigma=70 \mathrm{fs}$ ) is assumed to heat up the electron system, after which equilibration with the phonon and spin system will take place. On a longer time scale (picoseconds), heat will slowly diffuse away into the substrate, which is taken into account by heat flow from the electron system towards an infinite heat sink at ambient temperature. 
The magnetization dynamics (which is related to the spin temperature) can now be described as follows: ${ }^{27}$

$$
\frac{d m}{d t}=\frac{R T_{p}}{T_{C}}\left[m+b_{e f f}\right]\left[1-m \operatorname{coth}\left(\frac{T_{C}}{T_{e}}\left[m+b_{e f f}\right]\right)\right],
$$

where $m=\frac{\mathrm{M}}{\mathrm{M}_{\mathrm{s}}}$ is the magnetization relative to the saturation value, $T_{C}$ the Curie temperature, and $b_{\text {eff }}$ the effective magnetic field in the material normalized to the exchange field. $R\left(\mathrm{~s}^{-1}\right)$ is a prefactor in the demagnetization rate and equals $R=\frac{8 a_{s s} T_{C}^{2} g_{e p}}{k_{B} T_{D}^{2} D_{s}}$, with $a_{s f}$ the spin-flip rate, $g_{e p}$ the electron phonon coupling, $T_{D}$ the Debye temperature, and $D_{s}$ the atomic magnetic moment divided by $\mu_{B}$. Taking realistic parameters for $\mathrm{Co} / \mathrm{Pt}$ layers, ${ }^{30,31}$ we obtain $R=9.4 \mathrm{ps}^{-1}$. The effective magnetic field $b_{\text {eff }}$ is the sum of an external applied field and the magnetic field induced by the IFE. Due to this IFE, a polarized laser pulse can act as an effective magnetic field pulse. ${ }^{11}$ The induced magnetic field is modeled by a step function multiplied with an exponential decay with lifetime $\tau$, and convoluted with the Gaussian profile of the laser pulse. Both the strength and the (origin of the) lifetime of this IFE induced field pulse are still a subject of great controversy. Estimates for the strength range from 0.1 to $30 \mathrm{~T}$ for typical laser fluences and lifetimes from 0.1 to several ps are reported in literature..$^{3,11,24,26}$ Although both parameters can potentially be obtained from $a b$ initio calculations, or extracted by fitting experiments on similar systems (as we do for $R$ ), their exact values will depend on many details of the materials system and processes involved. Therefore, we investigate the influence of both parameters on the switching process by the proposed model, rather than fixing them to a single value.

Using the model as defined in the foregoing, we calculate the time evolution of the magnetization after excitation with a polarized laser pulse that induces a magnetic field via the IFE. This is done for three differently polarized laser pulses, a left- and right-handed circularly and linearly polarized pulse $\left(\sigma^{+}, \sigma^{-}, L\right.$, respectively). Typical results for our $\mathrm{Co} / \mathrm{Pt}$ model system, and assuming a long enough duration of the magnetic field pulse, are plotted in Figure 1. No magnetization reversal takes place in the case of L (no IFE) or $\sigma^{+}$(positive IFE) light, and only a rapid demagnetization and slower remagnetization is observed. However, for $\sigma^{-}$ (negative IFE) light a reversal of the magnetization is induced by the magnetic field pulse. We can thus model AOS with the M3TM if we invoke the IFE as the driving mechanism.

Since the lifetime is a crucial parameter in determining whether AOS will occur, its effect was previously investigated with an atomistic model. ${ }^{32}$ We have performed a similar investigation of the lifetime of the IFE induced field pulse using the M3TM, resulting in the phase diagram of Figure 2. For each set of parameters, it is determined whether the magnetization has switched $(\mathrm{M}<0)$ or not $(\mathrm{M}>0)$, or if the magnetization remains zero in the set simulation time of $20 \mathrm{ps}$. A switching window is observed that gets broader for longer lifetimes.

For low laser fluences, we do not see any switching as the magnetization is not yet sufficiently quenched. If the

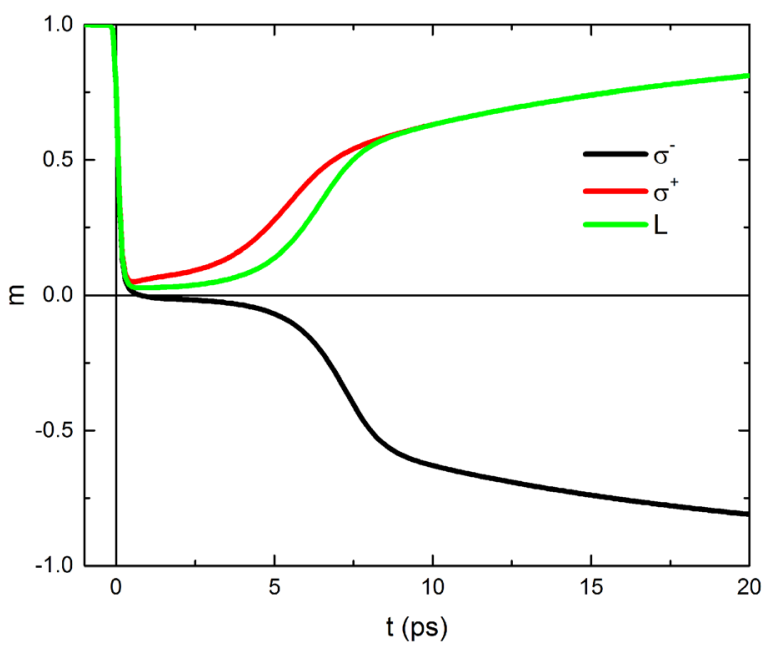

FIG. 1. The magnetization as a function of time after laser pulse excitation at $t=0$, for a left- and right-handed circularly and linearly polarized pulse $\left(\sigma^{+}, \sigma^{-}, L\right.$, respectively). The circularly polarized pulses are assumed to induce a magnetic field pulse with strength of $10 \mathrm{~T}$ and a lifetime of $0.4 \mathrm{ps}$; the linear pulse is assumed to induce no magnetic field.

fluence is increased, reversal becomes possible for long lifetimes. Further increasing the fluence, we see that only thermal demagnetization is observed. In that case, the sample is heated to such a degree that it cannot cool down in the set simulation time, resulting in zero final magnetization (unless a strong field is present). If the simulation is extended to a larger time scale, non-deterministic switching is observed in which numerical fluctuations determine the direction of the remagnetization. We see that a lifetime of at least $0.15 \mathrm{ps}$ is necessary to achieve AOS in this specific case. The actual lifetime of the IFE is still heavily debated, but there are claims that it could be above this required value. Alebrand et $a l^{3}$ even suggested that the helicity information might be stored for up to $100 \mathrm{ps}$, but did not link this directly to the IFE. Lifetimes as long as these would result in rather wide reversal windows. Even without such speculative dramatic effects, one can conclude that much shorter lifetimes may be enough for all-optical switching. More generally, it can be concluded that our phase diagram corresponds quite well

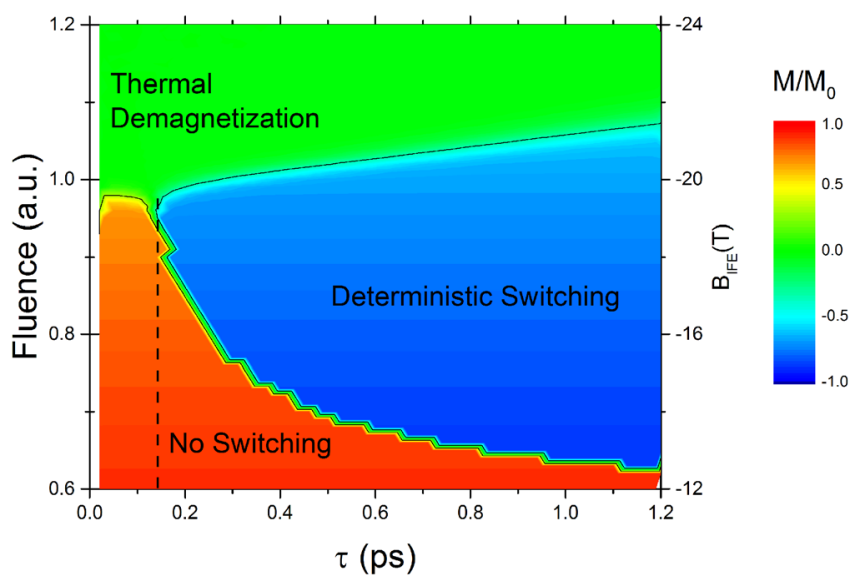

FIG. 2. Phase diagram of the final magnetization after excitation with a laser pulse with the given power fluence and induced field pulse lifetime $\tau$. The initial value of $\mathrm{M} / \mathrm{M}_{0}$ is +1 . The peak magnitude of the magnetic field pulse scales with the pulse power between 12 and $24 \mathrm{~T}$. The dashed line indicates the minimal lifetime needed to obtain switching. 
both qualitatively and quantitatively to the results previously obtained with the atomistic model. ${ }^{32}$ This confirms the validity of both models for describing AOS, where the M3TM has the benefit of being much faster.

The threshold for the lifetime turns out to be strongly dependent on specific simulation parameters. If the demagnetization rate is increased, the magnetization dynamics will speed up. One would expect that an even shorter lifetime is then sufficient for switching, which is indeed observed in the simulations (see Fig. S1). ${ }^{31}$ The minimal lifetime is found to decrease rapidly with $R$. For example, we note that increasing $R$ by just a factor of 1.5 to $15 \mathrm{ps}^{-1}$, a lifetime as short as $0.07 \mathrm{ps}$ is sufficient for switching, which is even below the laser pulse duration of $0.1 \mathrm{ps}$ (see Fig. S2). ${ }^{31}$ Previously, it has been assumed that a much longer lifetime was necessary for switching, which cast doubt on the relevance of the IFE in AOS. Therefore, the extremely short lifetime we now find makes the IFE a much more likely explanation for AOS. It also shows that enhancing the demagnetization rate increases the feasibility of AOS, which is of crucial importance for its application potential. We assign the shorter lifetime necessary to an enhanced spin-flip scattering at the $\mathrm{Co} /$ Pt interfaces as compared to, e.g., pristine Co films, as was experimentally demonstrated for Co/Pt multilayers. ${ }^{30}$ This notion is of particular interest in view of present activities on using materials with strong spin-orbit coupling for various storage and memory applications.

The above outcomes indicate that it should be possible to achieve AOS with circularly polarized pulses. However, in experiments non-local effects are involved that are not included in our macrospin M3TM discussed so far. Thus, we therefore add several spatial effects into the model by considering an array of $200 \times 200 \times 1 \mathrm{~nm}^{3}$ domains. We verified that the exact size of the domains only marginally affects our results. The Gaussian spatial profile of the laser pulse is taken into account and multiple pulse experiments are simulated by using the output of one simulation as starting conditions for the next. A Gaussian distributed magnetic field noise of $0.15 \mathrm{mT}$ is added to account for inhomogeneities in the sample and applied magnetic fields. Finally, the last ingredient included into our model is the effect of dipolar stray-fields, which have been suggested to play a role in AOS. ${ }^{13,22}$ The strength of the dipole fields can be calculated using standard electromagnetism equations, and is typically $0.6 \mathrm{mT}$ for nearest neighbors, when averaged over a complete cell. These stray fields will favor an antiparallel orientation between neighboring domains, thereby supporting the multi-domain state that is often observed after excitation with a high power laser pulse, usually referred to as thermal demagnetization. With these additions, we can fully model the images found in AOS experiments in ferromagnets. ${ }^{13}$

Starting with the simplest case, Figure 3 shows the magnetization profile after a single pulse obtained from simulations with this extended model (with no applied field), taking a magnetic field pulse strength and lifetime of $3 \mathrm{~T}$ and $0.3 \mathrm{ps}$, respectively. In the center of the laser spot, the sample gets heated to such a degree that all magnetic information is lost and a multi-domain state will form (thermal demagnetization). In a small outer circle region,

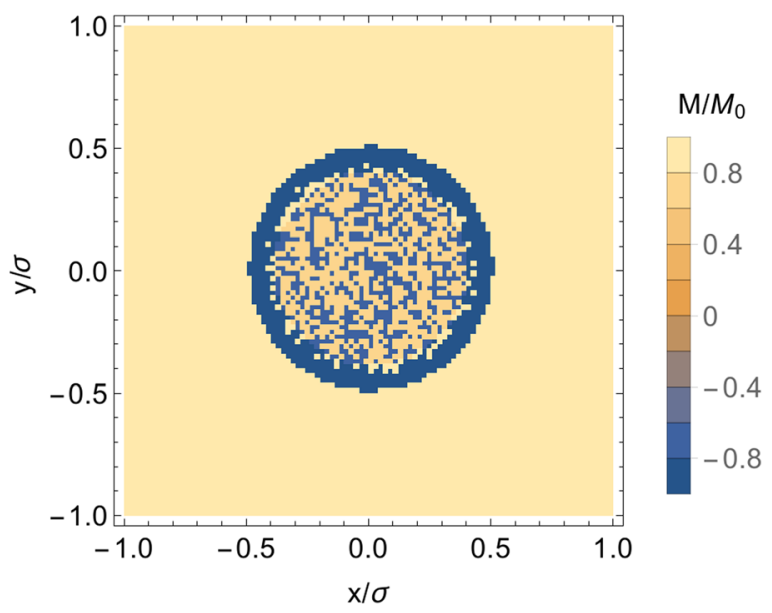

FIG. 3. Single pulse magnetization profile obtained by the M3TM simulation on a $[\mathrm{Co}(0.4 \mathrm{~nm}) / \operatorname{Pt}(0.7 \mathrm{~nm})]_{3}$ multilayer. The axes are scaled to the laser pulse spatial width $\boldsymbol{\sigma}$.

deterministic switching takes place, as is generally observed in AOS experiments. $2,6,13$

Having explained the effect of a single pulse with the extended model, we now look at the effect of a train of 12 pulses that is swept across the sample at a speed of $0.2 \mu \mathrm{m} /$ pulse, while applying a global magnetic field opposite to the IFE field. Figure 4(a) shows the magnetization profiles obtained from such simulations. For zero applied magnetic field, we see a typical result of an AOS experiment. The result is similar to Figure 3, but by sweeping multiple pulses across the sample a uniformly switched area is created. When the externally applied field is increased to $1.8 \mathrm{mT}$, we see that this field cancels the effect of the IFE. Non-deterministic switching now takes place and the uniformly switched area breaks apart into a multi-domain state. The multi-domain state at the center of the final pulse disappears due to the applied field. For an even higher applied field, no switching is observed at all. (a)

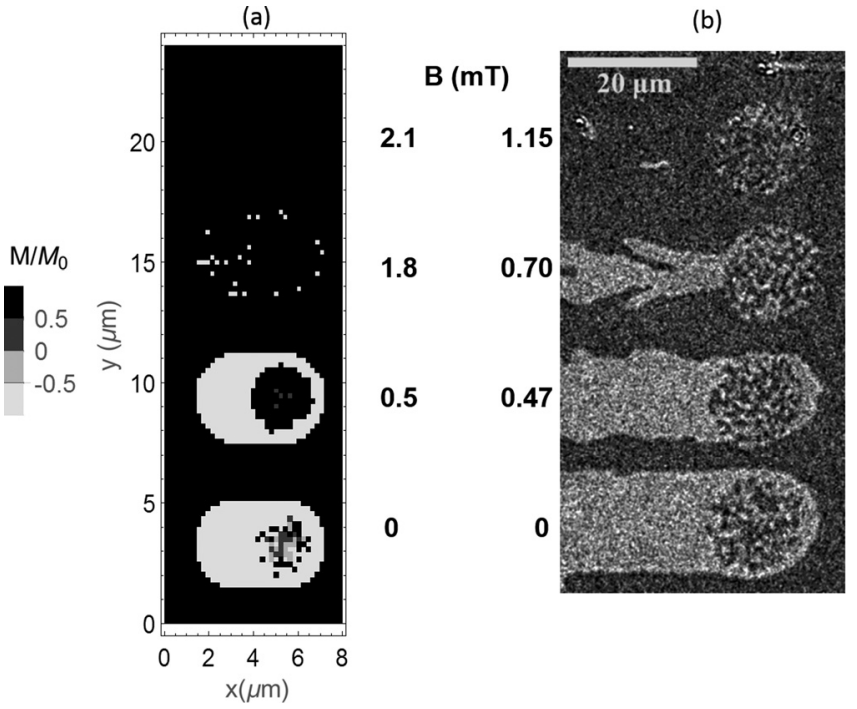

FIG. 4. Magnetization profiles, (a) obtained by the M3TM simulations and (b) as in the experiments of Lambert et al. ${ }^{13}$ on a $[\mathrm{Co}(0.4 \mathrm{~nm}) / \operatorname{Pt}(0.7 \mathrm{~nm})]_{3}$ multilayer. (Reproduced with permission from Lambert et al., Science $\mathbf{3 4 5}$, 1337 (2014). Copyright 2014, American Association for the Advance of Science.) Four situations with increasingly positive applied fields are shown. 
We now compare the results of the simulations to experimental results of Lambert et al., ${ }^{13}$ which is currently the only demonstration of AOS in ferromagnets. Figure 4(b) shows typical results on a $\mathrm{Co} / \mathrm{Pt}$ multilayer for an increasing counteracting applied field. Comparing the simulations with the experiments, we find a good overall agreement in the magnetization profiles. The applied fields, at which the transition from switching to no switching occurs, are of roughly the same magnitude. We note that this transition is dependent on the strength and lifetime of the IFE, and that the combination of the simulations and experiments therefore provides a way to assess these parameters. There are two main differences between the two results. The first is the disappearance of the thermal demagnetization in the center of the final laser pulse, whereas we see in the experiments that this thermal demagnetization persists even for high applied fields. The dipolar fields do support the formation of a multidomain state, but are not strong enough to fully explain the thermal demagnetization. It is likely that thermal activation, which is not included in the model, provides a way for domains to switch after remagnetization, thereby assisting the dipole fields in creating a multi-domain state. Second, we see that in the experiments large interconnected domains are formed when the applied field cancels the effect of the IFE. These large domains are formed due to domain wall dynamics, something that is not included in our simple model because it will have little effect on the ultrafast switching process itself.

To conclude, we have simulated AOS in ferromagnets using the M3TM. Using the IFE as the driving mechanism, the possibility of AOS was discussed. It was found that the parameters necessary for AOS are well within the current theoretical estimates. In particular, a minimal IFE lifetime below $0.1 \mathrm{ps}$ was obtained for material parameters close to $\mathrm{Co} / \mathrm{Pt}$ with strong spin-orbit coupling, which means that much shorter pulses may be needed than earlier claimed to be necessary. In addition, a real AOS experiment was simulated by adding spatial effects to the M3TM. The experimental magnetization profiles reported in $\mathrm{Co} / \mathrm{Pt}$ structures ${ }^{13}$ could be reproduced for realistic values of all simulation parameters. The effect of an applied field could also be reproduced. Hence, we found a good agreement with all experimental observations, showing that the IFE is a viable candidate to explain AOS in ferromagnetic systems like Co/ Pt indeed.

This work was supported by the Project No. FOM08.1898, a programme supported by the Dutch Government.

${ }^{1}$ E. Beaurepaire, J.-C. Merle, A. Daunois, and J. Y. Bigot, Phys. Rev. Lett. 76, 4250 (1996).

${ }^{2}$ C. D. Stanciu, F. Hansteen, A. V. Kimel, A. Kirilyuk, A. Tsukamoto, A. Itoh, and T. Rasing, Phys. Rev. Lett. 99, 047601 (2007).
${ }^{3}$ S. Alebrand, A. Hassdenteufel, D. Steil, M. Cinchetti, and M. Aeschlimann, Phys. Rev. B: Condens. Matter Mater. Phys. 85, 92401 (2012).

${ }^{4}$ A. Hassdenteufel, B. Hebler, C. Schubert, A. Liebig, M. Teich, M. Helm, M. Aeschlimann, M. Albrecht, and R. Bratschitsch, Adv. Mater. 25, 3122 (2013).

${ }^{5}$ A. Kirilyuk, A. V. Kimel, and T. Rasing, Rep. Prog. Phys. 76, 026501 (2013).

${ }^{6}$ S. Mangin, M. Gottwald, C.-H. Lambert, D. Steil, V. Uhliŕ, L. Pang, M. Hehn, S. Alebrand, M. Cinchetti, G. Malinowski, Y. Fainman, M. Aeschlimann, and E. E. Fullerton, Nat. Mater. 13, 286 (2014).

${ }^{7}$ I. Radu, K. Vahaplar, C. Stamm, T. Kachel, N. Pontius, H. A. Dürr, T. A. Ostler, J. Barker, R. F. L. Evans, R. W. Chantrell, A. Tsukamoto, A. Itoh, A. Kirilyuk, T. Rasing, and A. V. Kimel, Nature 472, 205 (2011).

${ }^{8}$ J. P. van der Ziel, P. S. Pershan, and L. D. Malmstrom, Phys. Rev. Lett. 15, 190 (1965).

${ }^{9}$ A. Kirilyuk, A. V. Kimel, and T. Rasing, Rev. Mod. Phys. 82, 2731 (2010).

${ }^{10}$ C. D. Stanciu, F. Hansteen, A. V. Kimel, A. Tsukamoto, A. Itoh, A. Kirilyuk, and T. Rasing, Phys. Rev. Lett. 98, 207401 (2007).

${ }^{11}$ A. V. Kimel, A. Kirilyuk, P. A. Usachev, R. V. Pisarev, A. M. Balbashov, and T. Rasing, Nature 435, 655 (2005).

${ }^{12}$ A. R. Khorsand, M. Savoini, A. Kirilyuk, A. V. Kimel, A. Tsukamoto, A. Itoh, and T. Rasing, Phys. Rev. Lett. 108, 127205 (2012).

${ }^{13}$ C.-H. Lambert, S. Mangin, B. S. D. C. S. Varaprasad, Y. K. Takahashi, M. Hehn, M. Cinchetti, G. Malinowski, K. Hono, Y. Fainman, M. Aeschlimann, and E. E. Fullerton, Science 345, 1337 (2014).

${ }^{14}$ R. Hertel, J. Magn. Magn. Mater. 303, L1 (2006).

${ }^{15}$ M. Battiato, G. Barbalinardo, and P. M. Oppeneer, Phys. Rev. B 89, 014413 (2014).

${ }^{16}$ M. I. Kurkin, N. B. Bakulina, and R. V. Pisarev, Phys. Rev. B 78, 134430 (2008).

${ }^{17}$ D. Hinzke, M. Berritta, U. Atxitia, R. Mondal, P. M. Oppeneer, and U. Nowak, in 2015 IEEE International Magnetics Conference (IEEE, 2015), pp. $1-1$.

${ }^{18}$ S. Wang, X. Li, J. Zhou, and M. Gu, Opt. Express 23, 13530 (2015).

${ }^{19}$ S. R. Woodford, Phys. Rev. B 79, 212412 (2009).

${ }^{20}$ D. D. Awschalom, J. Warnock, and S. von Molnár, Phys. Rev. Lett. 58, 812 (1987).

${ }^{21}$ T. Ogasawara, N. Iwata, Y. Murakami, H. Okamoto, and Y. Tokura, Appl. Phys. Lett. 94, 162507 (2009).

${ }^{22}$ L. Gierster, A. A. Ünal, L. Pape, F. Radu, and F. Kronast, Ultramicroscopy 159, 508 (2015).

${ }^{23}$ J. H. Mentink, J. Hellsvik, D. V. Afanasiev, B. A. Ivanov, A. Kirilyuk, A. V. Kimel, O. Eriksson, M. I. Katsnelson, and T. Rasing, Phys. Rev. Lett. 108, 057202 (2012).

${ }^{24}$ K. Vahaplar, A. M. Kalashnikova, A. V. Kimel, S. Gerlach, D. Hinzke, U. Nowak, R. Chantrell, A. Tsukamoto, A. Itoh, A. Kirilyuk, and T. Rasing, Phys. Rev. B 85, 104402 (2012).

${ }^{25}$ N. Kazantseva, D. Hinzke, U. Nowak, R. W. Chantrell, U. Atxitia, and O. Chubykalo-Fesenko, Phys. Rev. B 77, 184428 (2008).

${ }^{26}$ P. Nieves and O. Chubykalo-Fesenko, Phys. Rev. Appl. 5, 014006 (2016).

${ }^{27}$ B. Koopmans, G. Malinowski, F. Dalla Longa, D. Steiauf, M. Fähnle, T. Roth, M. Cinchetti, and M. Aeschlimann, Nat. Mater. 9, 259 (2010).

${ }^{28}$ T. Roth, A. J. Schellekens, S. Alebrand, O. Schmitt, D. Steil, B. Koopmans, M. Cinchetti, and M. Aeschlimann, Phys. Rev. X 2, 021006 (2012).

${ }^{29}$ A. J. Schellekens and B. Koopmans, Phys. Rev. B: Condens. Matter Mater. Phys. 87, 20407 (2013).

${ }^{30}$ K. C. Kuiper, T. Roth, A. J. Schellekens, O. Schmitt, B. Koopmans, M. Cinchetti, and M. Aeschlimann, Appl. Phys. Lett. 105, 202402 (2014).

${ }^{31}$ See supplementary material at http://dx.doi.org/10.1063/1.4945660 for details on the model parameters and the influence of the demagnetization rate $\mathrm{R}$.

${ }^{32}$ K. Vahaplar, A. M. Kalashnikova, A. V. Kimel, D. Hinzke, U. Nowak, R. W. Chantrell, A. Tsukamoto, A. Itoh, A. Kirilyuk, and T. Rasing, Phys. Rev. Lett. 103, 117201 (2009). 